\title{
Treatment with Number 2 Feibi Recipe, a Compound Traditional Chinese Medicine Reduces Bleomycin-induced Pulmonary Fibrosis in Mice
}

\author{
Qi Long", Zhao Heng Liu ${ }^{2}$, Jie Niu ${ }^{3}$, Hao Ge Liu ${ }^{1}$, Xiao Feng Gu ${ }^{1}$, Qing Lu Pang ${ }^{1}$, Fang Cao ${ }^{3}$, , \\ Yang Jiao ${ }^{3, *}$ \\ ${ }^{1}$ Graduate School, Beijing University of Chinese Medicine, Beijing, China \\ ${ }^{2}$ School of Life Sciences, Beijing University of Chinese Medicine, Beijing, China \\ ${ }^{3}$ Dongfang Affiliated Hospital, Beijing University of Chinese Medicine, Beijing, China
}

Email address:

caofang62593731@163.com (Fang Cao),yangjiao2013@sina.cn (Yang Jiao)

*Corresponding author

\section{To cite this article:}

Qi Long, Zhao Heng Liu, Jie Niu, Hao Ge Liu, Xiao Feng Gu, Qing Lu Pang, Fang Cao, Yang Jiao. Treatment with Number 2 Feibi Recipe, a Compound Traditional Chinese Medicine Reduces Bleomycin-induced Pulmonary Fibrosis in Mice. Clinical Medicine Research. Vol. 10, No. 3, 2021, pp. 73-83. doi: 10.11648/j.cmr.20211003.13

Received: April 15, 2021; Accepted: May 8, 2021; Published: May 14, 2021

\begin{abstract}
Objective: Oxidative stress with reactive oxygen species (ROS) generated from exogenous oxidants and pollutants exposure is involved in the pathogenesis of Idiopathic pulmonary fibrosis (IPF). Number 2 Feibi Recipe (Number 2 FBR) is a traditional Chinese herbal formula which can attenuate the lung injury induced by PM2.5. The present study is to explore the effect and mechanism of Number 2 FBR on bleomycin (BLM)-induced pulmonary fibrosis in C57BL/6Cnc mice. Method: Bleomycin-induced C57BL/6Cnc mice were treated with Number 2 FBR and Sulforphane for two weeks. HE and Masson trichrome staining were performed to evaluate pathological changes in lung tissues. The extent of lung fibrosis was evaluated with fibrosis scores, collagen volume fraction, and hydroxyproline concentration. Levels of SOD and 8-iso-PGF2 $\alpha$ in lung tissues were measured by using commercial assay kits. The levels of Nrf2, SOD, GSH-Px, and TGF- $\beta 1$ relative protein and mRNA in lung tissues were measured by real time PCR and Western blot respectively. Results: The results showed that Number 2 FBR ameliorated bleomycin-induced pathological changes, collagen deposition and significantly decreased fibrosis scores, collagen volume fraction, and hydroxyproline concentration in the mice lungs. Additionally, Number 2 FBR inhibited the expression of 8-iso-prostaglandin F2 $\alpha$ (8-iso-PGF2 $\alpha$ ) and transforming growth factor beta1 (TGF- $\beta 1$ ), and increased the expression of Nuclear Factor Erythroid 2-Related Factor 2 (Nrf2), superoxide dismutase (SOD) and Glutathione peroxidase (GSH-Px) in lung tissues. Conclusion: Number 2 FBR has an effect of anti-fibrosis by regulating the lung oxidants and antioxidants balance.
\end{abstract}

Keywords: Number 2 Feibi Recipe, Chinese Medicine, Pulmonary Fibrosis, Nrf2, Bleomycin, Mice

\section{Introduction}

Idiopathic pulmonary fibrosis (IPF) is a chronic, progressive, fatal, fibrotic interstitial lung disease with a median survival time between 3-5 years from diagnosis and limited therapeutic options [1]. Although the pathogenesis is not fully understood, a growing body of evidence has demonstrated that oxidative stress plays a significant role in IPF [2]. Oxidative stress is defined as an imbalance between oxidant production and antioxidant defence in favour of oxidants, that leads to cellular dysfunction and tissue damage [3]. Cigarette smoke [4] and multiple environmental insults such as silica [5], coal dust [6], asbestos fibers [7], radiation [8], and certain chemotherapeutic agents [9], which are considered as risk factors for pulmonary fibrosis, have also been found to trigger the production of the reactive oxygen species (ROS) include hydroxyl radical, hydrogen peroxide, and superoxide radical [10]. The excess ROS overwhelm antioxidant capacity to perturb the balance in this 
reduction-oxidation equilibrium, and eventually lead to oxidative stress of cells and tissues.

The promotion of pulmonary fibrosis by oxidative stress involves multiple mechanisms that include (i) causing extensive modifications and damage to macromolecules (e.g., DNA, lipids, proteins), and trigger the generation of peroxidation products (e.g., DNA adducts, lipid peroxides) further promoting the cycle of alveolar damage and abnormal repair [11-13], (ii) results in an acute accumulation of inflammatory cells in the alveolar spaces and interstitium through the activation of nuclear factor-kappa B (NF- $\mathrm{B})[14$, 15], (iii) promote alveolar epithelial cell apoptosis through mitochondrial-mediated intrinsic pathways or death receptor-mediated extrinsic pathways [16], (iv) directly contribute to profibrotic activation of TGF- $\beta$, initiating a positive feedback loop of TGF- $\beta 1$ activation with resultant persistent fibrosis $[17,18]$, (v) promote apoptosis resistance of myofibroblasts leading to their accumulation [19], (vi) activate matrix metalloproteinases (MMPs), increasing their transcription, deactivating proteases, and subsequent collagen deposition [20, 21].

Lung protection against oxidants is guaranteed by protective antioxidants such as intracellular and extracellular superoxide dismutases (SODs) and detoxification enzyme systems (e.,g., glutathione-S-transferases) to restore systemic redox balance and maintain homeostasis [22]. The induction of antioxidant enzymes and related proteins after exposure to insults may protect the lung and promote damage repair. Conversely, reduced induction or inactivation of antioxidant enzymes may result in a continuous redox imbalance, that may contribute to the progression of pulmonary fibrosis [23].

Nuclear factor erythroid-derived 2-like2 protein (Nrf2) is a key transcriptional regulator for antioxidant response element (ARE) -mediated induction of cellular antioxidant enzymes and detoxifying enzymes, which protects against the pathogenesis of pulmonary fibrosis [24, 25]. Indeed, deficiency of Nrf2 significantly enhanced bleomycin-induced pulmonary fibrosis in mice [26, 27]. Moreover, in IPF patients, compensatory increased pulmonary Nrf2 expression as well as elevated antioxidant levels in the broncho-alveolar fluid are reported [28]. Observations from these studies implicated that the Nrf2-antioxidant system may provide potential therapeutic strategies for IPF.

Traditional Chinese medicine (TCM) has more than two thousand year clinical practice history. Nowadays, TCM formulations are still widely used in pulmonary disorders in China. Number 2 Feibi Recipe (Number 2 FBR) is a traditional Chinese medicine formula according to Professor Ping'an Zhou's more than 50 years' clinical experience for treating pulmonary fibrosis. Former research approved that Number 2 FBR could decrease the expression of TGF- $\beta 1$, IL-6 in lung tissue, downregulate the expression of IL-6, IL-13, IL-17, MCP-1, TNF- $\alpha$, and upregulate GSH-Px in serum [29]. It suggested that the Number 2 FBR can inhibit fibrosis and immunoinflammatory injury, meanwhile enhancing protective antioxidant. Nevertheless, the mechanisms by which Number 2 FBR attenuate pulmonary fibrotic effects have not been fully elucidated. Based on our previous studies, we designed this study to evaluate the antifibrotic properties of Number 2 FBR and focused on exploring whether it enhances the Nrf2-antioxidant system to attenuate BLM-induced PF in mice.

\section{Materials and Methods}

\subsection{Reagents and Materials}

Bleomycin (lot No 6115292) was purchased from Fresenius Kabi. Sulforphane(lot No C4733) was purchased from APExBIO. Hydroxyproline (HYP) kit (lot No A030-2-1) was purchased from Nanjing Jiancheng Biochemical Institute. Modified masson's trichrome stain kit (lot No 20191108) was purchased from Beijing Solarbio Science technology Co. Ltd. Mouse 8-iso-PGF2 $\alpha$ ELISA Kit (lot No DG94697Q-96T), Mouse SOD ELISA Kit (lot No DG30430M-96T) were purchased from Dogesce. Nrf2 Antibody (Cat No bs-1074r), GSH-Px Antibody (Cat No bs-3882r), TGF- $\beta 1$ Antibody (Cat No bs-0086r) were purchased from Bioss.

\subsection{Preparation of No. 2 FBR}

Number 2 FBR was purchased from Beijing Tong Ren Tang, the Pharmaceutical Industry Co. The components of it are Radix Astragali, Rhodiolae Crenulatae Radix et Rhizoma, Flos Lonicerae Japonicae, Radix Scutellariae, Radix et Rhizoma Salviae Miltiorrhizae, Radix et Rhizoma Glycyrrhizae. Total net weight is $140 \mathrm{~g}$.

\subsection{Animals}

All animal experiments were approved by the Animal Research Ethics Board at Dongfang Hospital Affiliated to Beijing University of Chinese Medicine. A total of 40 C57BL/6Cnc male mice, weighing 20-24g, were purchased from Beijing Vital River Laboratory Animal Technology Co., Ltd. After 7 days adaptive feeding in community cages with $12 \mathrm{~h}$ periods of light and dark cycles and maintained on standard rodent chow with access to water ad libitum, all mice were randomly assigned into four groups ( $\mathrm{n}=10$ per group): Normal control group, Model group, Sulforphane group, and Number 2 FBR group.

The mice were inhalation anesthetized by propylene glycol isoflurane 2:3 mixture. Hung the teeth of anesthesia mice rapidly on a suspension line, kept the body erect, dragged out the tongue with curved pliers, dripped bleomycin $(5 \mathrm{mg} / \mathrm{kg}$, dissolved in sterile normal saline) quickly into the trachea, then rotated the mice to the left and right uprightly 3-5 times to make the bleomycin suspension evenly distributed in the lungs. Groups except for the normal control group were dripped bleomycin into the trachea. The normal control group was given equal amounts of normal saline.

Considered of the body surface area between human beings and mice, starting from the next day of model making, we gave Number 2 FBR $(10 \mathrm{ml} / \mathrm{kg})$ with the concentration of $2.102 \mathrm{~g} / \mathrm{ml}$ to the Number 2 FBR group by gavage for 14 days. Mice in Sulforphane group were intraperitoneally injected with Sulforphane $(10 \mathrm{ml} / \mathrm{kg})$ with the concentration of 
$2.5 \mathrm{mg} / \mathrm{ml}$ for 14 days. Normal control group and the model group were taken equal amounts of normal saline by gavage for 14 days.

\subsection{Sample Collection}

On the fifteenth day, the blood of the mice was drawn from the abdominal aorta under pentobarbital anesthesia and the lungs were removed. The left lungs were fixed by polyoxymethylene for HE staining, Masson's trichrome staining, and immunohistochemical staining, and the right lungs were frozen in liquid nitrogen for Western Blot and Real Time PCR.

\subsection{General Histological Staining}

The left lung was fixed in $10 \%$ formalin for more than $24 \mathrm{~h}$ and then dehydrated and embedded in paraffin. Coronal sections $(5 \mu \mathrm{m})$ of the upper, upper-mid, lower-mid, and lower part of the left lungs were deparaffinized and stained with HE and Masson's trichrome. Three consecutive but not repeated microscopic fields of each section were microscopically photographed with a 20 -fold magnification by a technician blinded to the results of animal grouping (twelve photographs per mouse). All photographs photographed sharply, $>95 \%$ of photographs had to be covered with lung tissue to ensure that the frequency of each grade of fibrosis was similar to avoid a bias to certain fibrotic grades.

One observer evaluated the HE staining photographs independently using the modified Ashcroft scale [45]. Areas with dominating tracheal or bronchial tissue were omitted. The grades were summarized to obtain a fibrotic index for the lung.

Masson's trichrome staining photographs were evaluated by Image $\mathrm{J} 1.52 \mathrm{i}$ (Wayne Rasband, NIH, USA). The gray-scale threshold count method was used to quantify the area occupied by collagen staining blue colour within each photograph. Collagen volume fraction $(\mathrm{CVF})$ was calculated based on the percent of blue collagen staining quantified within a tissue section. (Collagen volume fraction $(\%)=$ area of collagen fiber/area of total tissue) [46].

\subsection{Measurement of Content of HYP, SOD, and 8-iso-PGF2 $\alpha$ in Lung Tissues}

Hydroxyproline (HYP) content of lung tissue was measured by using hydroxyproline kits. SOD and 8 -iso-PGF $2 \alpha$ levels in lung tissues were measured by ELISA kits. All procedures were performed in accordance with the manufacturer's instructions.

\subsection{Western Blot Was Used in Analysis of Protein in Lung Tissue}

Levels of Nrf2, GSH-Px, and TGF- $\beta 1$ expression in full thickness were analyzed. The tissue was homogenized in RIPA lysis buffer. The homogenate was centrifuged at $12000 \mathrm{r} / \mathrm{min}$ (the centrifuge from THERMO, legend micro $21 \mathrm{r}$ ), for $10 \mathrm{~min}$, and the supernatant was collected. The protein samples were denatured by boiling for 5 minutes in
SDS sample buffer (MDL). Afterward, equal quantities of protein were separated with $10 \%$ SDS-PAGE and transferred to polyvinylidene difluoride (PVDF). The membranes were blocked with 5\% skim milk for 2 hours and then incubated with primary antibodies (Nrf2, GSH-Px, and TGF- $\beta 1$ ) overnight at $4^{\circ} \mathrm{C}$. After exposure to secondary antibodies for 60 minutes at $37^{\circ} \mathrm{C}$, the membranes were analyzed with ECL reagents. All assays were performed independently and repeated three times.

\subsection{Real Time PCR}

The tissue was homogenized in liquid nitrogen. Total RNA was extracted using a Transzol reagent. The total RNA concentration and purity were determined by UV-Vis spectroscopy using the Bio-Rad SmartSpec 5000 system (Bio-Rad, Hercules, CA, USA). cDNA reverse transcribe was according to the instructions of Super Script III RT Reverse Transcription kit (ABI-Invitrogen). The primers were designed (Table 1). $\beta$-actin was used as an endogenous control. The mRNA levels were examined by real-time PCR. A single melting curve peak confirmed the presence of a single product The mRNA level was quantified relative to $\beta$-actin using the 2- $\Delta \Delta \mathrm{CT}$ method.

Table 1. Sequences of primers used for the real-time PCR analysis.

\begin{tabular}{ll}
\hline Gene & Oligonucleotide primer sequences $\left(\mathbf{5}^{\prime}-\mathbf{3}^{\prime}\right)$ \\
\hline \multirow{2}{*}{ Nrf2 } & Forward: TCCCAGCAGGACATGGATTT \\
& Reverse: GGCCTTCTCCTGTTCCTTCT \\
SOD & Forward: CCCAGACCTGCCTTACGACT \\
& Reverse: TACTTCTCCTCGGTGGCGTT \\
GSH-Px & Forward: AACCTACGAGGGAGGAACAC \\
& Reverse: CTGGCAGAGACTGGGATCAA \\
TGF- $\beta 1$ & Forward: TTGCTTCAGCTCCACAGAGA \\
& Reverse: CAGAAGTTGGCATGGTAGCC \\
actin & Forward: CTCCTGAGCGCAAGTACTCT \\
& Reverse: TACTCCTGCTTGCTGATCCAC \\
\hline
\end{tabular}

\subsection{Statistical Analysis}

The data are expressed as mean \pm SD. Statistical testing was performed with SPSS software version 12.0 (SPSS Inc., Chicago, IL, USA). Data distribution was assessed using the D'Agostino-Pearson normality test. Parametric data was analyzed with a t-test for comparison of two variables and ANOVA with Tukey-Kramer post-test analysis for multiple comparisons. If the data did not satisfy the criteria for nonparametric homogeneity and normality, the Kruskal-Wallis test was used for comparison of two variables and Dunn's test for multiple comparisons. $P<0.05$ was considered statistically significant.

\section{Results}

\subsection{Histologically Evaluating of Pulmonary Fibrosis}

HE (Figure 1a) and Masson trichrome (Figure 1b) staining were performed to evaluate pathological changes in lung tissues. Compared with the normal control group, obvious alveolar structural disorder, abnormal thickening of the 
alveolar wall, massive deposition of matrix, amount of blue collagen fibers, and fibroblasts appeared in the model group after the BLM intratracheal instillation. Compared with the model group, the lung injury and fibrosis induced by BLM were reduced to varying degrees in both sulforphane and Number 2 FBR groups.

We performed a quantitative visual assessment of the degree of pulmonary fibrosis on HE staining photographs by the modified Ashcroft scale. The scores of fibrotic lesions were significantly increased 14 days after bleomycin administration, compared with those in the normal control group. However, after 14 days treatment of sulforphane or Number 2 FBR, scores were decreased compared with the model group. And the effect was more significant when treated with Number 2 FBR $(P<0.05)$ (Figure 1c)

We next performed a quantitative assessment of the degree of pulmonary fibrosis on Masson trichrome staining photographs. CVF was the parameter to be measured. Compared with the normal control group, CVFs were significantly higher in the model, suiforphane, and Number 2 FBR groups. However, CVFs in suiforphane and Number 2 FBR groups were decreased compared with the model group. And compared with model or suiforphane group, CVFs in Number 2 FBR group were lower with the statistical difference $(P<0.05)$. (Figure 1d)
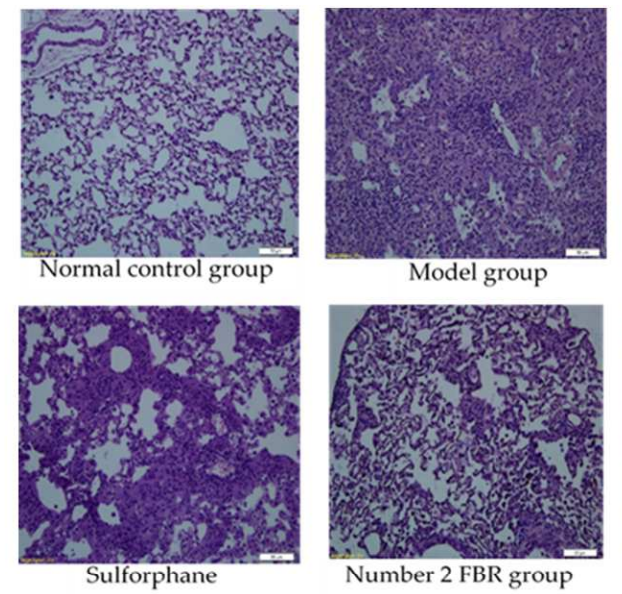

(a)

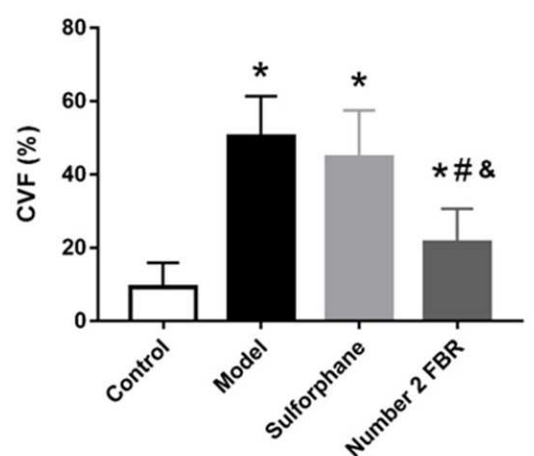

(c)

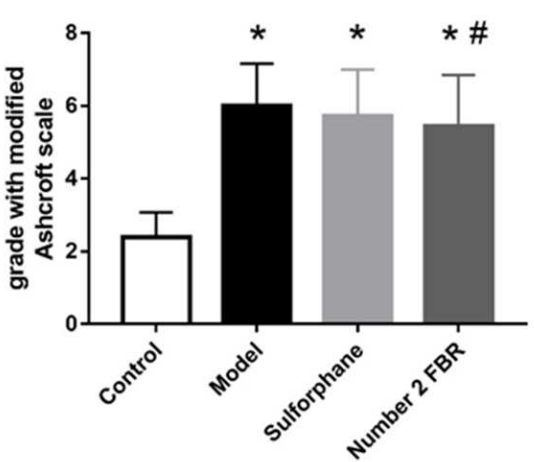

(d)
We further assessed the degree of pulmonary fibrosis by measuring the lung hydroxyproline content, which we found to be significantly increased 14 days after bleomycin administration compared with the normal control group. The concentration of hydroxyproline in the suiforphane and Number 2 FBR groups, however, were reduced compared with the model group. And compared with model or suiforphane group, the concentrations of hydroxyproline in Number 2 FBR group were lower with the statistical difference $(P<0.05)$ (Figure 1e).

\subsection{Expression of Nrf2, SOD, GSH-Px, and TGF-ß1 mRNAs in Lung Tissues}

Compared with the normal control group, the levels of $\mathrm{Nrf} 2$, SOD, GSH-Px, and TGF- $\beta 1 \mathrm{mRNAs}$ expression increased in the lung tissues after BLM administration. The levels of Nrf2, SOD, and GSH-Px mRNAs expression in the suiforphane group were significantly greater than the model group $(P<$ $0.05)$. The levels of SOD mRNA expression in Number 2 FBR group were significantly greater than the model group $(P<$ 0.05). The levels of TGF- $\beta 1 \mathrm{mRNA}$ expression in Number 2 FBR group were significantly decreased compared with the model group $(P<0.05)$ (Figure 2$)$.
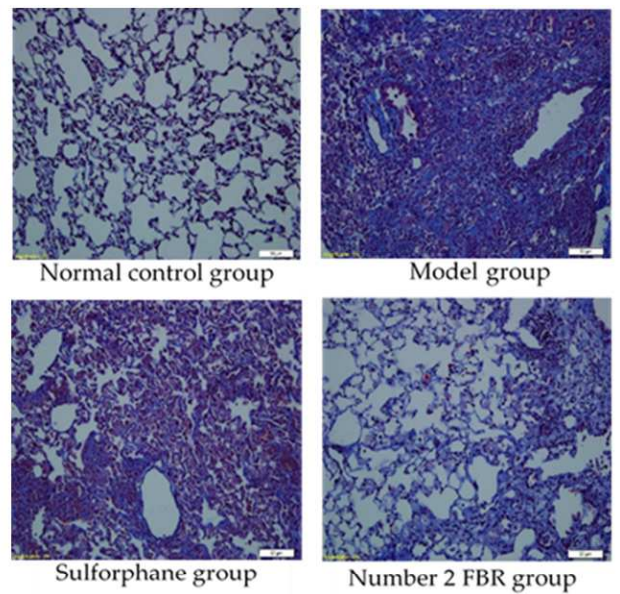

(b)
Number 2 FBR group

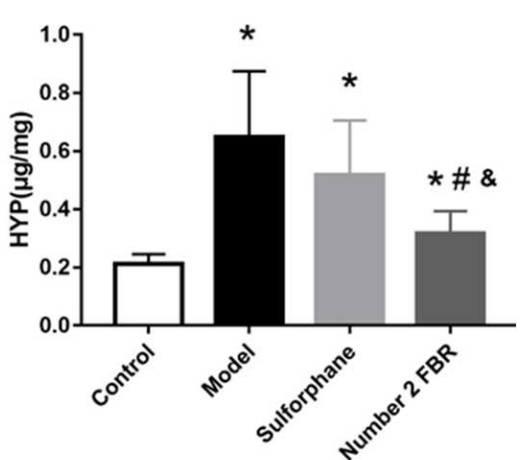

(e)

Figure 1. Effect of Number 2 FBR on histopathologic in BLM-induced pulmonary fibrosis mice. Representative photographs of sections stained with HE(a) and Masson trichrome (b) are shown at the same magnification $(200 \times)$. Quantitative assessment of pulmonary fibrosis with HE staining photographs $(c)$. $n=6$ in each group. quantitative assessment of Pulmonary fibrosis with Masson trichrome staining photographs (d). $n=6$ in each group. Hydroxyproline (HYP) content of lung tissues(e). $n=6$ in each group. Data were expressed as mean $\pm S . D$. * $P<0.05$ compared with the normal control group. \# $P<0.05$ compared with the model group. \& $P<0.05$ compared with the sulforphane group. 


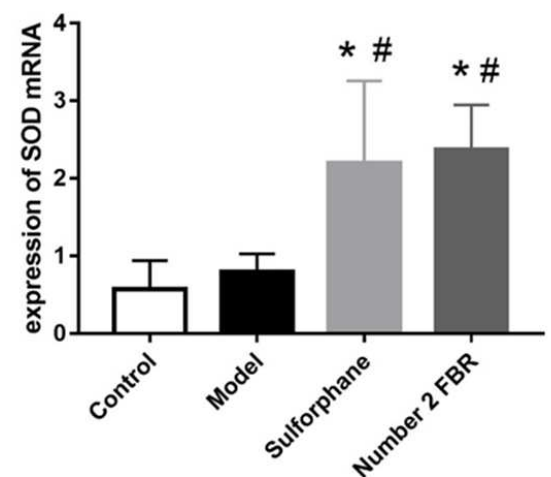

(a)

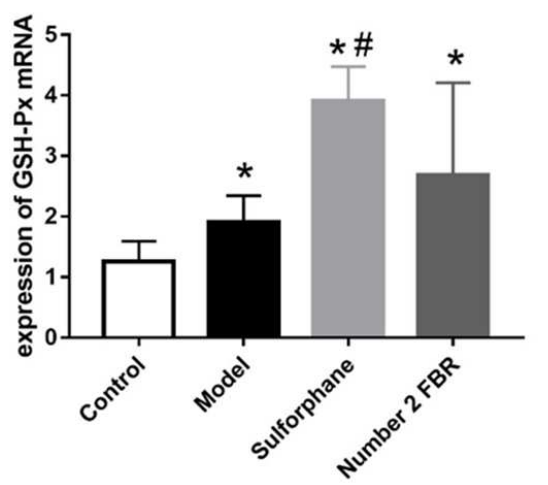

(c)

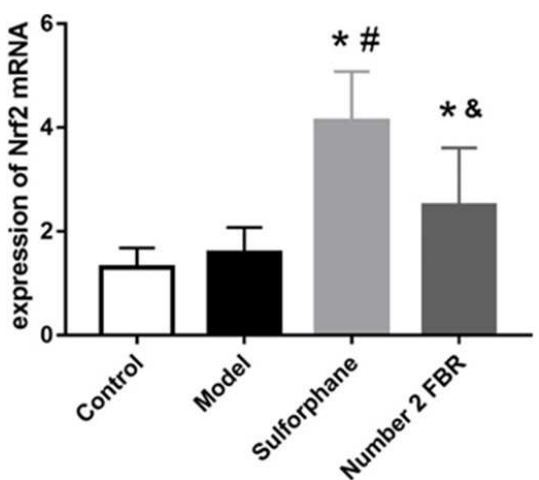

(b)

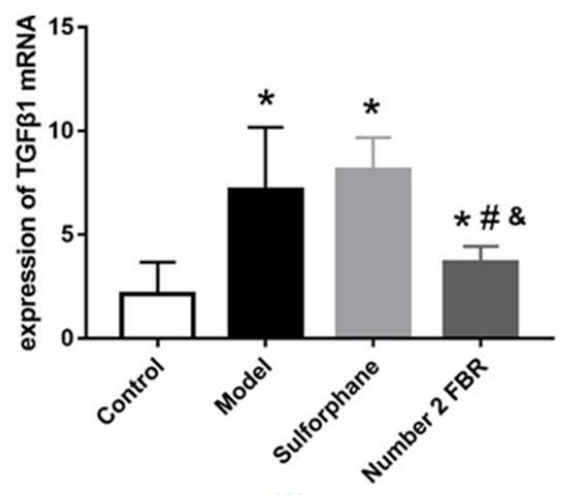

(d)

Figure 2. Effect of Number 2 FBR on the mRNA expressions of Nrf2, SOD, GSH-Px, and TGF- $\beta 1$ in the lung tissues of BLM-induced pulmonary fibrosis mice. The semiquantitative results of $N r f 2(a)$, $S O D(b), G S H-P x(c)$ and TGF- $\beta 1(d) m R N A$ expression in the lung tissues. $n=3$ in each group. Data were expressed as mean \pm S.D. $* P<0.05$ compared with the normal control group. $\# P<0.05$ compared with the model group. \& $P<0.05$ compared with the sulforphane group.

Control Model Sulfoephane Number 2 FBR

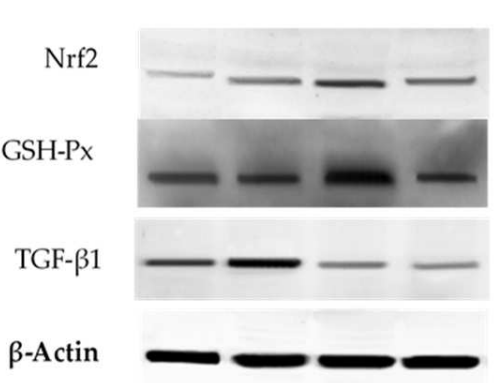

(a)

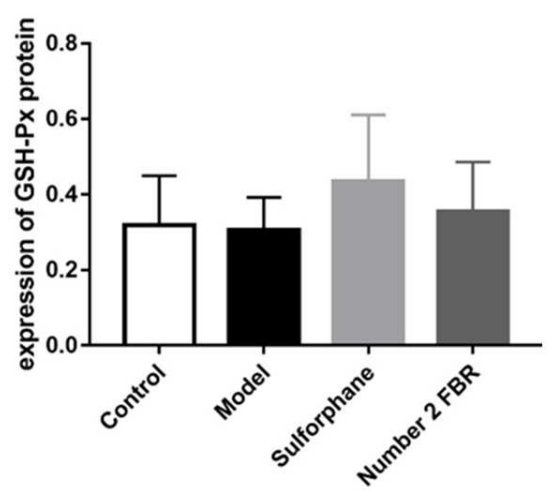

(c)

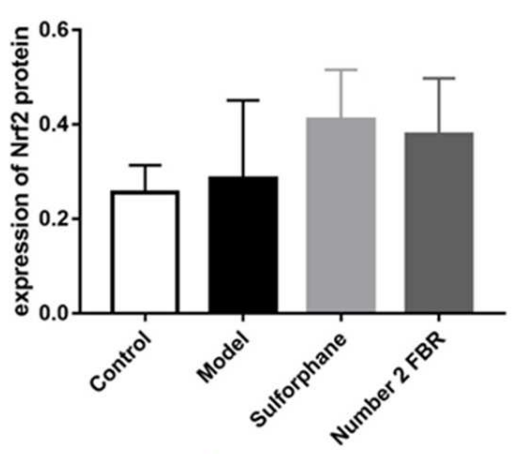

(b)

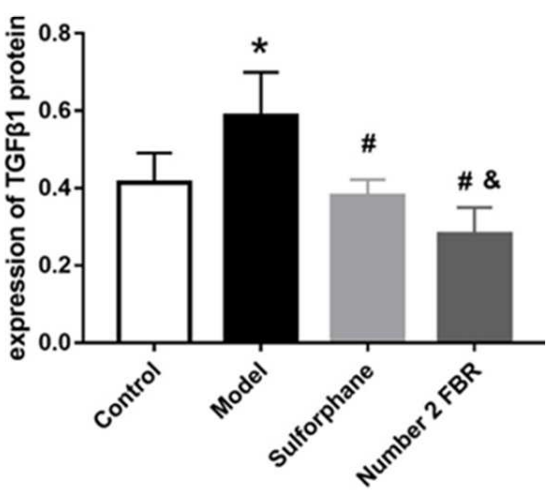

(d)

Figure 3. Effect of Number 2 FBR on the protein expressions of Nrf2, GSH-Px, and TGF- 1 1 in the lung tissues of BLM-induced pulmonary fibrosis mice. The $N r f 2, G S H-P x$, and TGF- 1 protein expressions in the lung tissues were detected by Western blot analysis(a). The quantitative results of the Nrf2(b), GSH-Px(c) and TGF- $\beta 1(d)$ protein expressions. $n=3$ in each group. Data were expressed as mean $\pm S . D$. * $P<0.05$ compared with the normal control group. \# $P<0.05$ compared with the model group. \& $P<0.05$ compared with the sulforphane group. 


\subsection{Effect of Number 2 FBR on Levels of the Protein of Nrf2, GSH-Px, and TGF-p1 in Lung Tissues}

Compared with the normal control group, the level of TGF- $\beta 1$ protein expression significantly increased in the model group after BLM administration $(P<0.05)$. However, after 14 days treatment of sulforphane or Number 2 FBR, the level of TGF- $\beta 1$ protein significantly decreased compared with the model group $(P<0.05)$. Furthermore, there was no statistical significance comparing the level of Nrf2 and GSH-Px in the 4 groups $(P>0.05)$. However, Number 2 FBR still had the trend to increase the level of Nrf2 and GSH-Px protein expression. The content of SOD and

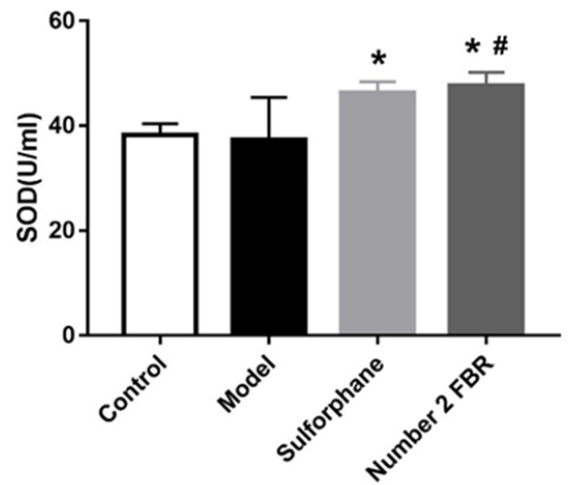

(a)
8-iso-PGF $2 \alpha$ in the lung tissues were measured by ELISA analysis (Figure 3 ).

\subsection{Effect of Number 2 FBR on Levels of SOD and 8-iso-PGF2a in Lung Tissues}

The content of SOD and 8-iso-PGF2 $\alpha$ in the lung tissues were measured by ELISA analysis. Compared with the normal control group, the levels of SOD were lower, and 8-iso-PGF2 $\alpha$ were higher in the model group. However, after 14 days treatment of Number 2 FBR, the content of SOD were significantly increased, and 8-iso-PGF2 $\alpha$ were significantly reduced compared with the model group $(P<0.05)$ (Figure 4$)$.

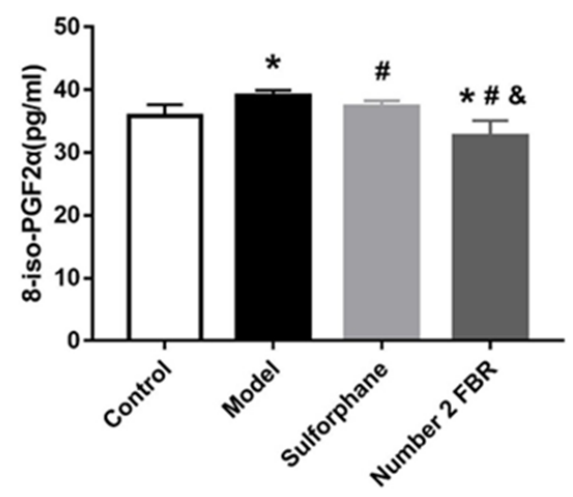

(b)

Figure 4. Effect of Number 2 FBR on the content of SOD and 8-iso-PGF2a in the lung tissues of BLM-induced pulmonary fibrosis mice. SOD (a) and 8 -iso-PGF2 $\alpha$ (b) content in the lung tissues detected by ELISA. $n=6$ in each group. Data were expressed as mean \pm S.D. $* P<0.05$ compared with the normal control group. \# $P<0.05$ compared with the model group. \& $P<0.05$ compared with the sulforphane group.

\section{Discussion}

In this study, we demonstrated that the Number 2 FBR has the effect of alleviating BLM-induced pulmonary fibrosis. A reduction was observed after the treatment of Number 2 FBR in hydroxyproline content of lung tissues, consistent with the decrease of visual assessment scores and collagen volume fraction in histological changes induced by bleomycin. The increase in the expression of SOD and GSH-Px in mRNA and protein levels, and the decrease of the concentration of 8 -iso-PGF $2 \alpha$ in lung tissues after Number 2 FBR administration indicates the beneficial role of Number 2 FBR to restore the redox imbalance induced by bleomycin. Furthermore, Number 2 FBR upregulated Nrf2 and decreased the expression of TGF- $\beta 1$ in mRNA and protein levels, suggesting the involvement of Nrf2 and TGF- $\beta 1$ in the signaling pathway for Number 2 FBR action.

Oxidative stress presents as a critical scenario in the progress of IPF. However, reactive oxygen species with a half-life of only milliseconds are almost unable to be detected directly in vivo [32]. In this present study, 8-iso-PGF2 $\alpha$, a widely accepted biomarker of oxidative stress [33], was detected to assess the extent of oxidative stress, and it was found that the concentration of 8 -iso-PGF2 $\alpha$ in lung tissue was significantly increased, the TGF- $\beta 1$ expression levels were elevated as well, and the fibrosis progressed, indicating that oxidative stress and persistent pulmonary fibrosis was induced as expected after bleomycin administration.

In the presence of bleomycin, the expression of Nrf2, SOD and GSH-Px in the lung tissues increased compensatively in mRNA and protein levels, but it was not sufficient to eliminate oxidative damage. After the treatment of Number 2 FBR, a clear reduction of 8 -iso-PGF $2 \alpha$ concentration and the significantly increasing of the SOD mRNA and protein expression were observed in lung tissues. In line with SOD and GSH-Px, Nrf2, the upstream regulator for antioxidant enzymes were also elevated, indicating that Number 2 FBR could confer protection against bleomycin induced oxidative stress through activating Nrf2 signaling pathway to promote the expression of antioxidant enzymes. In addition, the inappropriate activation of TGF- $\beta 1$ and subsequent fibrosis progression, which has been proved to be initiated by ROS [34], were attenuated in lung tissues of Number 2 FBR-treated mice, as evidenced by significantly reduced TGF- $\beta 1$ expression, hydroxyproline content, collagen volume fraction, and histopathological scores.

In this study, sulforaphane, a strong Nrf2 activator which has been shown to activate antioxidant response [35], was used as positive control. After 14 days intraperitoneally injection of sulforaphane, the expression of Nrf2, SOD, GSH-Px in lung tissues were upregulated, 8-iso-PGF2 $\alpha$ concentration of lung tissues were decreased, which indicates the effect of sulforaphane on the Nrf2 signaling pathway 
activation and the induction of downstream antioxidant enzymes to reduce oxidative stress. But the hydroxyproline content, collagen volume fraction, and histopathological scores were only have a reduced trend but without statistically significant after sulforaphane treatment, which shows that the anti-fibrosis effect of sulforaphane was not satisfactory.

Table 2. Pulmonary fibrosis related biological functions of ingredients isolated from Number 2 FBR components.

\begin{tabular}{|c|c|c|c|}
\hline Components & ingredients & Biological Functions associated with PF & References \\
\hline & Polysaccharides & Inhibit EMT and NF-кB pathway & [47] \\
\hline & & Inhibit TGF- $\beta 1 / \mathrm{Smad} 3$ pathway & [37] \\
\hline Radix & Astragaloside IV & Attenuate ECM deposition & [36] \\
\hline \multirow[t]{2}{*}{ Astragali } & & Inhibit EMT & {$[38,39]$} \\
\hline & & Anti-oxidative, anti-inflammatory & [48] \\
\hline Flos Lonicerae Japonicae & Chlorogenic Acids & Anti-oxidative, anti-inflammatory & {$[49,50]$} \\
\hline Radix & Baicalein & inhibit miR-21 & {$[45]$} \\
\hline Scutellariae & & upregulate $\mathrm{A} 2 \mathrm{aR}$ & [43] \\
\hline Rhodiolae & Gallic acid & Anti-oxidative & {$[51]$} \\
\hline Crenulatae & & Inhibit EMT & {$[52]$} \\
\hline Radix et & & Anti-inflammation & {$[53]$} \\
\hline \multirow[t]{3}{*}{ Rhizoma } & Rosavin & Inhibit NF- $\kappa B$ p 65 , TGF- $\beta 1$ and $\alpha-S M A$ & {$[54]$} \\
\hline & & Inhibit TGF- $\beta$ pathway & {$[55]$} \\
\hline & Salvianolic acid B & Inhibit myofibroblast trans differentiation & {$[56]$} \\
\hline Rhizoma & & decrease TNF- $\alpha$ and TGF- $\beta 1$, increase PGE2 & [59] \\
\hline Salviae & & Anti-oxidative, anti-inflammatory & {$[57,60-63]$} \\
\hline \multirow[t]{5}{*}{ Miltiorrhizae } & Tanshinone IIA & inhibit TGF $\beta$-dependent EMT & [41] \\
\hline & & inhibit TGF- $\beta 1$-Smad pathway & {$[40,62]$} \\
\hline & & modulate ACE-2/ANG-(1-7) axis & [42] \\
\hline & cryptotanshinone & inhibit Smad and STAT3 pathways & {$[64,65]$} \\
\hline & Protocatechuic aldehyde & modulate HMGB1/RAGE pathway & {$[66]$} \\
\hline Radix et & & Inhibit TGF $\beta$, EMT and fibroblast proliferation & [67] \\
\hline Rhizoma Glycyrrhizae & Glycyrrhizic acid & anti-inflammatory, anti-oxidative, inhibit TGF $\beta 1 / \operatorname{Smad} 2$ pathway and NOX4 & [68] \\
\hline
\end{tabular}

Interestingly, compared with sulforaphane, Number 2 FBR activated Nrf2 single signaling pathway to lesser extents, but showed obvious advantages in terms of the effective in suppressing TGF- $\beta 1$ activation and fibrosis progression. Combined with the anti-inflammatory effect of Number 2 FBR have been found in our previous experiments, it was hypothesized that the effect of Number 2 FBR for ameliorating pulmonary fibrosis was involved in some other mechanisms besides improving oxidative stress. The results of pharmacological studies confirmed this hypothesis by that the active ingredients of Number 2 FBR components attenuate pulmonary fibrosis through multiple mechanisms (Table 2). For example, in addition to the antioxidant function, Astragaloside IV extracted from Radix Astragali was shown to inhibit epithelial-mesenchymal transition (EMT) and TGF- $\beta 1 /$ Smad3 pathway and attenuating extracellular matrix (ECM) deposition [36-39], and Tanshinone IIA from Radix et Rhizoma Salviae Miltiorrhizae was shown to inhibit TGF- $\beta 1-S m a d$ pathway and EMT, and modulating ACE-2/ANG-(1-7) axis [40-42]. Moreover, the main active ingredients of Radix Scutellariae, Baicalin and Baicalein, could alleviate fibroblast proliferation, prevent pulmonary fibrosis through inhibiting $\mathrm{miR}-21$ and upregulating Adenosine A2a receptor (A2aR) [43-45].

IPF is a highly complex disease and involves multiple genes and processes. Previous studies have shown that purely oxidant scavenging has not been enough to completely cure or prevent IPF [46], and our findings of the sulforaphane group in this study are consistent with this concept. In contrast, as a compound preparation, Number 2 FBR alleviate the fibrosis process in a more delicate way by acting on a variety of fibrosis related targets which including but not limited to oxidative stress might be more effective to exert beneficial effects on pulmonary fibrosis.

Number 2 FBR consists of six different components, each of them contains numerous bioactive ingredients, and the mechanisms of its mitigation of pulmonary fibrosis is far from fully understood. The present study focused on the effect of Number 2 FBR as a whole on the BLM-induced pulmonary fibrosis and its antioxidant stress mechanism. Much more work is required to elucidate other underlying mechanisms and the role of any individual ingredient in Number 2 FBR in the effect observed.

\section{Conclusions}

This study demonstrated that Number 2 FBR has an effect on protecting against the development of pulmonary fibrosis and might be employed as a therapeutic candidate agent for attenuating pulmonary fibrosis. An involvement of $\mathrm{Nrf} 2$ in the signaling pathway responsible for the protective effect of Number 2 FBR on the oxidative and antioxidant balance of bleomycin-induced pulmonary fibrosis.

The evidence from the present study also suggested Number 2 FBR alleviate the fibrosis process by acting on a variety of fibrosis related targets. There are some underlying mechanisms required to elucidate. 


\section{Abbreviations}

\begin{tabular}{|c|c|}
\hline ROS & reactive oxygen species \\
\hline IPF & idiopathic pulmonary fibrosis \\
\hline $\begin{array}{l}\text { Number } 2 \\
\text { FBR }\end{array}$ & Number 2 Feibi Recipe \\
\hline BLM & bleomycin \\
\hline 8 -iso-PGF2 $\alpha$ & 8-iso-prostaglandin F2 $\alpha$ \\
\hline TGF- $\beta 1$ & transforming growth factor beta 1 \\
\hline Nrf2 & Nuclear Factor Erythroid 2-Related Factor 2 \\
\hline SOD & superoxide dismutase \\
\hline GSH-Px & glutathione peroxidase \\
\hline $\mathrm{NF}-\kappa \mathrm{B}$ & nuclear factor-kappa B \\
\hline MMP & matrix metalloproteinase \\
\hline ARE & antioxidant response element \\
\hline TCM & traditional Chinese medicine \\
\hline $\mathrm{MCP}$ & monocyte chemoattractant protein \\
\hline IL-6 & interleukin-6 \\
\hline IL-13 & interleukin-13 \\
\hline IL-17 & interleukin-17 \\
\hline TNF- $\alpha$ & tumor necrosis factor- $\alpha$ \\
\hline HYP & hydroxyproline \\
\hline CVF & collagen volume fraction \\
\hline ECM & extracellular matrix \\
\hline EMT & epithelial-mesenchymal transition \\
\hline $\mathrm{PF}$ & pulmonary fibrosis \\
\hline $\mathrm{A} 2 \mathrm{aR}$ & adenosine $\mathrm{A} 2 \mathrm{a}$ receptor \\
\hline PGE2 & prostaglandin E2 \\
\hline ACE-2 & angiotensin-converting enzyme 2 \\
\hline ANG-(1-7) & angiotensin-(1-7) \\
\hline HMGB1 & high mobility group box 1 \\
\hline RAGE & receptor for advanced glycation end-product \\
\hline STAT3 & $\begin{array}{l}\text { signal transducer and activator of } \\
\text { transcription }\end{array}$ \\
\hline NOX4 & NADPH oxidase 4 \\
\hline
\end{tabular}

\section{Author Contributions}

Qi Long and Yang Jiao conceived and designed the research; Qi Long, Haoge Liu, Xiaofeng $\mathrm{Gu}$, and Qinglu Pang performed the experiments; Qi Long and Zhaoheng Liu analyzed the data; Jie Niu, and Fang Cao contributed reagents/materials/analysis tools; Qi Long wrote the first draft of the manuscript and Yang Jiao participated in revision. All authors have read and agreed to the published version of the manuscript.

\section{Conflicts of Interest}

The authors declare that they have no competing interests.

\section{Funding}

This research was funded by Beijing Natural Science Foundation, grant number 7202118 and National Natural
Science Foundation of China, grant number 81573970.

\section{References}

[1] Richeldi, L.; Collard, H. R.; Jones, M. G., Idiopathic pulmonary fibrosis. Lancet 2017, 389, (10082), 1941-1952.

[2] Cheresh, P.; Kim, S. J.; Tulasiram, S.; Kamp, D. W., Oxidative stress and pulmonary fibrosis. Biochim Biophys Acta 2013, $1832,(7), 1028-40$.

[3] Fois, A. G.; Paliogiannis, P.; Sotgia, S.; Mangoni, A. A.; Zinellu, E.; Pirina, P.; Carru, C.; Zinellu, A., Evaluation of oxidative stress biomarkers in idiopathic pulmonary fibrosis and therapeutic applications: a systematic review. Respir Res 2018, 19, (1), 51.

[4] Lederer, D. J.; Martinez, F. J., Idiopathic Pulmonary Fibrosis. $N$ Engl J Med 2018, 378, (19), 1811-1823.

[5] Baumgartner, K. B.; Samet, J. M.; Coultas, D. B.; Stidley, C. A.; Hunt, W. C.; Colby, T. V.; Waldron, J. A., Occupational and environmental risk factors for idiopathic pulmonary fibrosis: a multicenter case-control study. Collaborating Centers. Am J Epidemiol 2000, 152, (4), 307-15.

[6] Vanhee, D.; Gosset, P.; Wallaert, B.; Voisin, C.; Tonnel, A. B., Mechanisms of fibrosis in coal workers' pneumoconiosis. Increased production of platelet-derived growth factor, insulin-like growth factor type I, and transforming growth factor beta and relationship to disease severity. Am J Respir Crit Care Med 1994, 150, (4), 1049-55.

[7] Barber, C. M.; Fishwick, D., Idiopathic pulmonary fibrosis and asbestos use. Bmj 2019, 364, 11041.

[8] Abid, S. H.; Malhotra, V.; Perry, M. C., Radiation-induced and chemotherapy-induced pulmonary injury. Curr Opin Oncol 2001, 13, (4), 242-8.

[9] Raghu, G.; Collard, H. R.; Egan, J. J.; Martinez, F. J.; Behr, J.; Brown, K. K.; Colby, T. V.; Cordier, J. F.; Flaherty, K. R.; Lasky, J. A.; Lynch, D. A.; Ryu, J. H.; Swigris, J. J.; Wells, A. U.; Ancochea, J.; Bouros, D.; Carvalho, C.; Costabel, U.; Ebina, M.; Hansell, D. M.; Johkoh, T.; Kim, D. S.; King, T. E., Jr.; Kondoh, Y.; Myers, J.; Müller, N. L.; Nicholson, A. G.; Richeldi, L.; Selman, M.; Dudden, R. F.; Griss, B. S.; Protzko, S. L.; Schünemann, H. J., An official ATS/ERS/JRS/ALAT statement: idiopathic pulmonary fibrosis: evidence-based guidelines for diagnosis and management. Am J Respir Crit Care Med 2011, 183, (6), 788-824.

[10] Walters, G. I., Occupational exposures and idiopathic pulmonary fibrosis. Curr Opin Allergy Clin Immunol 2020, 20, (2), 103-111.

[11] Gladyshev, V. N., The free radical theory of aging is dead. Long live the damage theory! Antioxid Redox Signal 2014, 20, (4), 727-31.

[12] Kanoh, S.; Kobayashi, H.; Motoyoshi, K., Exhaled ethane: an in vivo biomarker of lipid peroxidation in interstitial lung diseases. Chest 2005, 128, (4), 2387-92.

[13] Hogg, J. C., Why does airway inflammation persist after the smoking stops? Thorax 2006, 61, (2), 96-7. 
[14] Adamson, I. Y.; Bowden, D. H., Role of polymorphonuclear leukocytes in silica-induced pulmonary fibrosis. Am J Pathol 1984, 117, (1), 37-43.

[15] Cheng, N.; Shi, X.; Ye, J.; Castranova, V.; Chen, F.; Leonard, S. S.; Vallyathan, V.; Rojanasakul, Y., Role of transcription factor NF-kappaB in asbestos-induced TNFalpha response from macrophages. Exp Mol Pathol 1999, 66, (3), 201-10.

[16] Circu, M. L.; Aw, T. Y., Reactive oxygen species, cellular redox systems, and apoptosis. Free Radic Biol Med 2010, 48, (6), 749-62.

[17] Pociask, D. A.; Sime, P. J.; Brody, A. R., Asbestos-derived reactive oxygen species activate TGF-beta1. Lab Invest 2004, $84,(8), 1013-23$.

[18] Card, J. W.; Racz, W. J.; Brien, J. F.; Massey, T. E., Attenuation of amiodarone-induced pulmonary fibrosis by vitamin $\mathrm{E}$ is associated with suppression of transforming growth factor-betal gene expression but not prevention of mitochondrial dysfunction. J Pharmacol Exp Ther 2003, 304, (1), 277-83

[19] Hecker, L.; Vittal, R.; Jones, T.; Jagirdar, R.; Luckhardt, T. R.; Horowitz, J. C.; Pennathur, S.; Martinez, F. J.; Thannickal, V. J., NADPH oxidase-4 mediates myofibroblast activation and fibrogenic responses to lung injury. Nat Med 2009, 15, (9), 1077-81.

[20] McKeown, S.; Richter, A. G.; O'Kane, C.; McAuley, D. F.; Thickett, D. R., MMP expression and abnormal lung permeability are important determinants of outcome in IPF. Eur Respir J 2009, 33, (1), 77-84.

[21] Tan, R. J.; Fattman, C. L.; Niehouse, L. M.; Tobolewski, J. M.; Hanford, L. E.; Li, Q.; Monzon, F. A.; Parks, W. C.; Oury, T. D., Matrix metalloproteinases promote inflammation and fibrosis in asbestos-induced lung injury in mice. Am J Respir Cell Mol Biol 2006, 35, (3), 289-97.

[22] Cameli, P.; Carleo, A.; Bergantini, L.; Landi, C.; Prasse, A.; Bargagli, E., Oxidant/Antioxidant Disequilibrium in Idiopathic Pulmonary Fibrosis Pathogenesis. Inflammation 2020, 43, (1), $1-7$.

[23] Kliment, C. R.; Oury, T. D., Oxidative stress, extracellular matrix targets, and idiopathic pulmonary fibrosis. Free Radic Biol Med 2010, 49, (5), 707-17.

[24] Suzuki, T.; Yamamoto, M., Molecular basis of the Keap1-Nrf2 system. Free Radic Biol Med 2015, 88, (Pt B), 93-100.

[25] Bellezza, I.; Giambanco, I.; Minelli, A.; Donato, R., Nrf2-Keap1 signaling in oxidative and reductive stress. Biochim Biophys Acta Mol Cell Res 2018, 1865, (5), 721-733.

[26] Kikuchi, N.; Ishii, Y.; Morishima, Y.; Yageta, Y.; Haraguchi, N.; Itoh, K.; Yamamoto, M.; Hizawa, N., Nrf2 protects against pulmonary fibrosis by regulating the lung oxidant level and Th1/Th2 balance. Respir Res 2010, 11, (1), 31.

[27] Walters, D. M.; Cho, H. Y.; Kleeberger, S. R., Oxidative stress and antioxidants in the pathogenesis of pulmonary fibrosis: a potential role for Nrf2. Antioxid Redox Signal 2008, 10, (2), 321-32.

[28] Markart, P.; Luboeinski, T.; Korfei, M.; Schmidt, R.; Wygrecka, M.; Mahavadi, P.; Mayer, K.; Wilhelm, J.; Seeger, W.; Guenther, A.; Ruppert, C., Alveolar oxidative stress is associated with elevated levels of nonenzymatic low-molecular-weight antioxidants in patients with different forms of chronic fibrosing interstitial lung diseases. Antioxid Redox Signal 2009, $11,(2), 227-40$

[29] Liu, Z.; Wang, W.; Cao, F.; Liu, S.; Zou, X.; Li, G.; Yang, H.; Jiao, Y., Number 2 Feibi Recipe Reduces PM2.5-Induced Lung Injury in Rats. Evid Based Complement Alternat Med 2018, 2018,3674145 .

[30] Hübner, R. H.; Gitter, W.; El Mokhtari, N. E.; Mathiak, M.; Both, M.; Bolte, H.; Freitag-Wolf, S.; Bewig, B., Standardized quantification of pulmonary fibrosis in histological samples. Biotechniques 2008, 44, (4), 507-11, 514-7.

[31] Yamamura, K.; Yuen, D.; Hickey, E. J.; He, X.; Chaturvedi, R. R.; Friedberg, M. K.; Grosse-Wortmann, L.; Hanneman, K.; Billia, F.; Farkouh, M. E.; Wald, R. M., Right ventricular fibrosis is associated with cardiac remodelling after pulmonary valve replacement. Heart 2019, 105, (11), 855-863.

[32] Egashira, T.; Takayama, F.; Yamanaka, Y., [Detection and characterization of free radicals, radical scavenging activity, and lipid peroxides in cerebral ischemia-reperfusion injury by electron spin resonance and chemiluminescence high-performance liquid chromatography]. Nihon Shinkei Seishin Yakurigaku Zasshi 1997, 17, (4), 153-8.

[33] Van't Erve, T. J.; Lih, F. B.; Jelsema, C.; Deterding, L. J.; Eling, T. E.; Mason, R. P.; Kadiiska, M. B., Reinterpreting the best biomarker of oxidative stress: The 8-iso-prostaglandin F2 $\alpha$ /prostaglandin F2 $\alpha$ ratio shows complex origins of lipid peroxidation biomarkers in animal models. Free Radic Biol Med 2016, 95, 65-73.

[34] Cui, Y.; Robertson, J.; Maharaj, S.; Waldhauser, L.; Niu, J.; Wang, J.; Farkas, L.; Kolb, M.; Gauldie, J., Oxidative stress contributes to the induction and persistence of TGF- $\beta 1$ induced pulmonary fibrosis. Int J Biochem Cell Biol 2011, 43, (8), 1122-33.

[35] Houghton, C. A.; Fassett, R. G.; Coombes, J. S., Sulforaphane and Other Nutrigenomic Nrf2 Activators: Can the Clinician's Expectation Be Matched by the Reality? Oxid Med Cell Longev 2016, 2016, 7857186.

[36] Li, L. C.; Xu, L.; Hu, Y.; Cui, W. J.; Cui, W. H.; Zhou, W. C.; Kan, L. D., Astragaloside IV Improves Bleomycin-Induced Pulmonary Fibrosis in Rats by Attenuating Extracellular Matrix Deposition. Front Pharmacol 2017, 8, 513.

[37] Li, N.; Feng, F.; Wu, K.; Zhang, H.; Zhang, W.; Wang, W., Inhibitory effects of astragaloside IV on silica-induced pulmonary fibrosis via inactivating TGF- $\beta 1 / \mathrm{Smad} 3$ signaling. Biomed Pharmacother 2019, 119, 109387.

[38] Qian, W.; Cai, X.; Qian, Q., Sirt1 antisense long non-coding RNA attenuates pulmonary fibrosis through sirt1-mediated epithelial-mesenchymal transition. Aging (Albany NY) 2020, $12,(5), 4322-4336$.

[39] Qian, W.; Cai, X.; Qian, Q.; Zhang, W.; Wang, D., Astragaloside IV modulates TGF- $\beta 1$-dependent epithelial-mesenchymal transition in bleomycin-induced pulmonary fibrosis. J Cell Mol Med 2018, 22, (9), 4354-4365.

[40] Feng, F.; Li, N.; Cheng, P.; Zhang, H.; Wang, H.; Wang, Y.; Wang, W., Tanshinone IIA attenuates silica-induced pulmonary fibrosis via inhibition of TGF- $\beta 1$-Smad signaling pathway. Biomed Pharmacother 2020, 121, 109586. 
[41] Tang, H.; He, H.; Ji, H.; Gao, L.; Mao, J.; Liu, J.; Lin, H.; Wu, T., Tanshinone IIA ameliorates bleomycin-induced pulmonary fibrosis and inhibits transforming growth factor-beta- $\beta$-dependent epithelial to mesenchymal transition. $J$ Surg Res 2015, 197, (1), 167-75.

[42] Wu, H.; Li, Y.; Wang, Y.; Xu, D.; Li, C.; Liu, M.; Sun, X.; Li, Z., Tanshinone IIA attenuates bleomycin-induced pulmonary fibrosis via modulating angiotensin-converting enzyme 2/ angiotensin-(1-7) axis in rats. Int $J$ Med Sci 2014, 11, (6), 578-86.

[43] Huang, X.; He, Y.; Chen, Y.; Wu, P.; Gui, D.; Cai, H.; Chen, A.; Chen, M.; Dai, C.; Yao, D.; Wang, L., Baicalin attenuates bleomycin-induced pulmonary fibrosis via adenosine A2a receptor related TGF- $\beta 1$-induced ERK1/2 signaling pathway. BMC Pulm Med 2016, 16, (1), 132.

[44] Zhao, H.; Li, C.; Li, L.; Liu, J.; Gao, Y.; Mu, K.; Chen, D.; Lu, A.; Ren, Y.; Li, Z., Baicalin alleviates bleomycin-induced pulmonary fibrosis and fibroblast proliferation in rats via the PI3K/AKT signaling pathway. Mol Med Rep 2020, 21, (6), 2321-2334.

[45] Gao, Y.; Lu, J.; Zhang, Y.; Chen, Y.; Gu, Z.; Jiang, X., Baicalein attenuates bleomycin-induced pulmonary fibrosis in rats through inhibition of miR-21. Pulm Pharmacol Ther 2013, 26, (6), 649-54.

[46] Canestaro, W. J.; Forrester, S. H.; Raghu, G.; Ho, L.; Devine, B. E., Drug Treatment of Idiopathic Pulmonary Fibrosis: Systematic Review and Network Meta-Analysis. Chest 2016, 149, (3), 756-66.

[47] Zhang, R.; Xu, L.; An, X.; Sui, X.; Lin, S., Astragalus polysaccharides attenuate pulmonary fibrosis by inhibiting the epithelial-mesenchymal transition and NF- $\mathrm{BB}$ pathway activation. Int J Mol Med 2020, 46, (1), 331-339.

[48] Yu, W. N.; Sun, L. F.; Yang, H., Inhibitory Effects of Astragaloside IV on Bleomycin-Induced Pulmonary Fibrosis in Rats Via Attenuation of Oxidative Stress and Inflammation. Inflammation 2016, 39, (5), 1835-41.

[49] Clifford, M. N.; Jaganath, I. B.; Ludwig, I. A.; Crozier, A., Chlorogenic acids and the acyl-quinic acids: discovery, biosynthesis, bioavailability and bioactivity. Nat Prod Rep 2017, 34, (12), 1391-1421.

[50] Liang, N.; Kitts, D. D., Role of Chlorogenic Acids in Controlling Oxidative and Inflammatory Stress Conditions. Nutrients 2015, 8, (1).

[51] Nikbakht, J.; Hemmati, A. A.; Arzi, A.; Mansouri, M. T.; Rezaie, A.; Ghafourian, M., Protective effect of gallic acid against bleomycin-induced pulmonary fibrosis in rats. Pharmacol Rep 2015, 67, (6), 1061-7.

[52] Jin, L.; Piao, Z. H.; Sun, S.; Liu, B.; Ryu, Y.; Choi, S. Y.; Kim, G. R.; Kim, H. S.; Kee, H. J.; Jeong, M. H., Gallic acid attenuates pulmonary fibrosis in a mouse model of transverse aortic contraction-induced heart failure. Vascul Pharmacol 2017, 99, 74-82.

[53] Rong, Y.; Cao, B.; Liu, B.; Li, W.; Chen, Y.; Chen, H.; Liu, Y.; Liu, T., A novel Gallic acid derivative attenuates BLM-induced pulmonary fibrosis in mice. Int Immunopharmacol 2018, 64, 183-191.

[54] Xin, X.; Yao, D.; Zhang, K.; Han, S.; Liu, D.; Wang, H.; Liu, X.;
Li, G.; Huang, J.; Wang, J., Protective effects of Rosavin on bleomycin-induced pulmonary fibrosis via suppressing fibrotic and inflammatory signaling pathways in mice. Biomed Pharmacother 2019, 115, 108870.

[55] Liu, Q.; Chu, H.; Ma, Y.; Wu, T.; Qian, F.; Ren, X.; Tu, W.; Zhou, X.; Jin, L.; Wu, W.; Wang, J., Salvianolic Acid B Attenuates Experimental Pulmonary Fibrosis through Inhibition of the TGF- $\beta$ Signaling Pathway. Sci Rep 2016, 6, 27610 .

[56] Liu, M.; Xu, H.; Zhang, L.; Zhang, C.; Yang, L.; Ma, E.; Liu, L.; Li, Y., Salvianolic acid B inhibits myofibroblast transdifferentiation in experimental pulmonary fibrosis via the up-regulation of Nrf2. Biochem Biophys Res Commun 2018, 495, (1), 325-331.

[57] Jiang, L.; Wang, J.; Ju, J.; Dai, J., Salvianolic acid B and sodium tanshinone II A sulfonate prevent pulmonary fibrosis through anti-inflammatory and anti-fibrotic process. Eur $J$ Pharmacol 2020, 883, 173352.

[58] Larki, A.; Hemmati, A. A.; Arzi, A.; Borujerdnia, M. G.; Esmaeilzadeh, S.; Zad Karami, M. R., Regulatory effect of caffeic acid phenethyl ester on type I collagen and interferon-gamma in bleomycin-induced pulmonary fibrosis in rat. Res Pharm Sci 2013, 8, (4), 243-52.

[59] Larki-Harchegani, A.; Hemmati, A. A.; Arzi, A.; Ghafurian-Boroojerdnia, M.; Shabib, S.; Zadkarami, M. R.; Esmaeilzadeh, S., Evaluation of the Effects of Caffeic Acid Phenethyl Ester on Prostaglandin E2 and Two Key Cytokines Involved in Bleomycin-induced Pulmonary Fibrosis. Iran $J$ Basic Med Sci 2013, 16, (7), 850-7.

[60] An, L.; Peng, L. Y.; Sun, N. Y.; Yang, Y. L.; Zhang, X. W.; Li, B.; Liu, B. L.; Li, P.; Chen, J., Tanshinone IIA Activates Nuclear Factor-Erythroid 2-Related Factor 2 to Restrain Pulmonary Fibrosis via Regulation of Redox Homeostasis and Glutaminolysis. Antioxid Redox Signal 2019, 30, (15), 1831-1848.

[61] Zhu, Z.; Li, Q.; Xu, C.; Zhao, J.; Li, S.; Wang, Y.; Tian, L., Sodium tanshinone IIA sulfonate attenuates silica-induced pulmonary fibrosis in rats via activation of the $\mathrm{Nrf} 2$ and thioredoxin system. Environ Toxicol Pharmacol 2020, 80, 103461 .

[62] Feng, F.; Cheng, P.; Xu, S.; Li, N.; Wang, H.; Zhang, Y.; Wang, W., Tanshinone IIA attenuates silica-induced pulmonary fibrosis via Nrf2-mediated inhibition of EMT and TGF- $\beta 1 /$ Smad signaling. Chem Biol Interact 2020, 319, 109024.

[63] He, H.; Tang, H.; Gao, L.; Wu, Y.; Feng, Z.; Lin, H.; Wu, T., Tanshinone IIA attenuates bleomycin-induced pulmonary fibrosis in rats. Mol Med Rep 2015, 11, (6), 4190-6.

[64] Zhang, Q.; Gan, C.; Liu, H.; Wang, L.; Li, Y.; Tan, Z.; You, J.; Yao, Y.; Xie, Y.; Yin, W.; Ye, T., Cryptotanshinone reverses the epithelial-mesenchymal transformation process and attenuates bleomycin-induced pulmonary fibrosis. Phytother Res 2020, $34,(10), 2685-2696$.

[65] Zhang, Y.; Lu, W.; Zhang, X.; Lu, J.; Xu, S.; Chen, S.; Zhong, Z.; Zhou, T.; Wang, Q.; Chen, J.; Liu, P., Cryptotanshinone protects against pulmonary fibrosis through inhibiting Smad and STAT3 signaling pathways. Pharmacol Res 2019, 147, 104307. 
[66] Zhang, L.; Ji, Y.; Kang, Z.; Lv, C.; Jiang, W., Protocatechuic aldehyde ameliorates experimental pulmonary fibrosis by modulating HMGB1/RAGE pathway. Toxicol Appl Pharmacol 2015, 283, (1), 50-6.

[67] Gao, L.; Tang, H.; He, H.; Liu, J.; Mao, J.; Ji, H.; Lin, H.; Wu, T., Glycyrrhizic acid alleviates bleomycin-induced pulmonary fibrosis in rats. Front Pharmacol 2015, 6, 215.
[68] Zhang, D.; Liu, B.; Cao, B.; Wei, F.; Yu, X.; Li, G. F.; Chen, H.; Wei, L. Q.; Wang, P. L., Synergistic protection of Schizandrin B and Glycyrrhizic acid against bleomycin-induced pulmonary fibrosis by inhibiting TGF- $\beta 1 / \mathrm{Smad} 2$ pathways and overexpression of NOX4. Int Immunopharmacol 2017, 48, $67-75$. 